Astudillo-Torres, M.P. \& Chévez-Ponce, F. (2021). Análisis del rol del docente universitario a partir de una crisis sanitaria: el proceso de una resignificación de lo presencial a lo virtual. Revista Electrónica Interuniversitaria de Formación del Profesorado, 24(2),139-151.

\title{
Análisis del rol del docente universitario a partir de una crisis sanitaria: el proceso de una resignificación de lo presencial a lo virtual
}

\author{
Martha Patricia Astudillo Torres', Florlenis Chévez Ponce ${ }^{2}$ \\ ${ }^{1}$ Universidad Autónoma de Chiapas, ${ }^{2}$ Universidad de Costa Rica
}

\section{Resumen}

La presente investigación analiza el rol del docente, en el contexto de educación superior, considerando los cambios acontecidos por la contingencia de la enfermedad Covid-19, la cual obligó a proseguir el proceso educativo de forma virtual en todo el mundo. El contexto del estudio se circunscribe en dos universidades públicas latinoamericanas ubicadas en México y Costa Rica; la investigación se desarrolla desde la metodología de la Teoría Fundamentada, integrando la entrevista en profundidad como técnica de recogida de información. El análisis de los datos se basa en el método comparativo constante, considerando el primer momento como descriptivo, el cual permite la codificación abierta y en el segundo momento, el relacional, que permite la codificación axial y selectiva generando una matriz de codificación. Los principales hallazgos explican el rol del docente en procura de responder a las urgentes medidas adoptadas por las instituciones educativas, en la que el educador debe enmarcar el proceso educativo desde una virtualidad total para el desarrollo de los contenidos.

\section{Palabras clave}

Pandemia; COVID-19; rol docente; TIC, virtualidad

\section{Contacto:}




\title{
Analysis of the role of the university teacher from a health crisis: the process of a resignification from the face-to-face to the virtual
}

\begin{abstract}
The present investigation analyzes the role of the teacher, in the context of higher education, considering the changes occurred by the contingency of the Covid-19 disease, which forced to continue the educational process virtually throughout the world. The context of the study is circumscribed in two Latin American public universities located in Mexico and Costa Rica; The research is developed from the methodology of the grounded theory, integrating indepth interview as a technique for collecting information. The data analysis is based on the constant comparative method, considering the first moment as descriptive, which allows open coding and the second, relational, which allows selective and axial coding, generating a coding matrix. The main findings seek to explain the role of the teacher in trying to respond to the urgent measures of educational institutions, in which the teacher must be framed in the educational process from a total virtuality for the development of content.
\end{abstract}

\section{Key words}

Pandemic; COVID-19; teaching role; ICT, virtuality

\section{Introducción}

Derivado de la contingencia originada por la crisis sanitaria debido a la Covid-19, el mundo prácticamente se paralizó, ocasionando que la mayoría de los ámbitos -económico, político y social- entraran en un letargo sin un final claro. Es así, que por disposición oficial se limita el acercamiento y socialización de las personas en sitios públicos y privados con base en dos clasificaciones relacionadas con las actividades esenciales y no esenciales. Dentro de las actividades esenciales se encuentran las que son directamente necesarias para atender la emergencia sanitaria, tales como las involucradas en la seguridad pública y la protección ciudadana y las de los sectores fundamentales de la economía: financieros, recaudación tributaria, distribución y venta de energéticos, gasolineras y gas, generación y distribución de agua potable, industria de alimentos y bebidas no alcohólicas, mercados de alimentos, además de las necesarias para la conservación, mantenimiento y reparación de la infraestructura crítica que asegura la producción y distribución de servicios indispensables $(D O F, 2020)$. Por otro lado, las actividades no esenciales abarcan todos los demás rubros no considerados en la primera clasificación, que incluye el sector educativo.

A partir de este panorama, las escuelas de todos los niveles han sido cerradas desde marzo de 2020 en México y Costa Rica y serán las últimas en reaperturar sus puertas para recibir a millones de profesores, estudiantes, directivos y demás trabajadores de apoyo. Al cerrar los centros educativos, producto de la crisis sanitaria, se evidencian costos en todos los sectores de la sociedad, es decir, un impacto sin precedente, que se genera en la educación, ya que las interrupciones hacen evidente las disparidades que se presentan dentro del sistema educativo. La Unesco (2020) señala que en la educación, se observan aspectos de transición de forma desordenada y frustrante, desventajas desproporcionadas para los estudiantes de mayor vulnerabilidad, el papel del docente muestra inseguridad sobre sus obligaciones y cómo llevar el apoyo a los estudiantes y padres de familia que no están preparados para guiar a sus hijos en este proceso de educación a distancia; encima de que 
los educadores deben plantear estrategias para llevar a cabo las evaluaciones a distancia, donde el panorama del acceso al aprendizaje es variable.

En este sentido, los profesores universitarios se vieron obligados a transferir de forma abrupta y no planificada las clases que impartían de forma presencial, a una modalidad completamente virtual para continuar con el proceso de enseñanza y aprendizaje, lo cual ha originado una serie aciertos y desaciertos para los agentes de la educación. Por ello, en esta investigación se analiza el rol docente universitario, indagando sobre elementos que han sido agregados a partir de su inmersión total a la virtualidad para poder llevar a cabo su quehacer.

El contexto del estudio se desarrolla en dos instituciones públicas de educación superior ubicadas en México y Costa Rica, para el cual se diseña un modelo metodológico guiado por los supuestos de la Teoría fundamentada, desde la perspectiva cualitativa. De este modo, se conoce de viva voz de los docentes sus interpretaciones relacionadas con el objeto de investigación, construyéndose desde lo que indican los actores: un proceso de resignificación de su papel. La relevancia de este estudio radica en que, desde los participantes, se puedan encontrar relaciones sobre las experiencias que han enfrentado para la entrada al espacio virtual en la impartición de las lecciones, pasando de la presencialidad que ha sido generalizada en la mayor parte de los sistemas educativos, a uno donde los aspectos virtuales median el proceso educativo.

\section{Marco teórico}

\subsection{Las TIC como base en el proceso educativo virtual durante la contingencia}

La situación educativa mundial provocada por la pandemia no tiene precedente, en décadas, puesto que ha obligado a las instituciones educativas a cerrar indefinidamente sus instalaciones, ocasionando, cambios drásticos en la forma de impartir la enseñanza y el aprendizaje. Desde este suceso, la comunidad científica se ha abocado al estudio del virus SARS-CoV-2, causante de la enfermedad COVID-19, desde diferentes disciplinas. Por ello, en este apartado presentaremos el posicionamiento de diversos estudios enfocados en el campo de la educación, llevados a cabo particularmente en la educación superior.

De acuerdo con Hodges, Moore, Lockee, Trust y Bond (2020) la experiencia de aprendizaje remoto en tiempos de crisis es un cambio temporal para establecer un proceso educativo alternativo, de manera que lo fundamental en esta situación no es pretender un ambiente robusto educativo, por el contrario, lo que se debe establecer son accesos temporales a los procesos de instrucción y apoyos educativos que sean flexibles y de mayor rapidez para configurar un espacio en el que se desarrollen los contenidos durante la crisis.

Por su parte, Brítez (2020) manifiesta que ante el cambio de política de la modalidad de la educación, Paraguay, Argentina y Brasil han tomado las medidas necesarias para no perder el año escolar, pero esto ha sacado a luz aspectos importantes a mejorar para impartir clases a distancia haciendo uso de los entornos virtuales de aprendizaje; dichos aspectos están relacionados con la falta de capacitación docente en herramientas tecnológicas WhatsApp, videoconferencias, conversores de archivos, Classroom- para continuar con el proceso formativo online, lo que, también, provoca falta de preparación de contenidos para ser compartidos en ambientes virtuales de aprendizaje. De igual manera, se presenta dificultad para realizar videoconferencias con la participación de los estudiantes y hacer retroalimentación con ellos.

Otro elemento que toma en cuenta este autor, son las carreras técnicas con actividades que no pueden realizarse de manera virtual y que derivan en sinnúmero de dificultades que van sucediendo todos los días, incluyendo el aspecto económico para el 
pago de las cuotas que ha generado un nuevo fenómeno denominado "paro virtual académico". A este respecto, son los estudiantes los que se han movilizado para resistir la transición digital, tal es el caso de la principal asociación estudiantil de Túnez que llamó a boicotear las plataformas digitales por considerar discriminatoria la medida. Los alumnos de la Universidad de Chile y de la Universidad de San Sebastián, realizaron huelgas en línea. Además, en el Reino Unido, más de 200,000 estudiantes firmaron una petición exigiendo rembolsos de sus pagos de matrícula, señalando que la instrucción por internet no era por lo que habían pagado (Alcántara, 2020).

En cuanto a la evaluación online, García, Corell, Avella y Grande (2020) señalan que esta representa una situación que las universidades presenciales no había enfrentado nunca, desde una perspectiva institucional en la que el profesorado y el estudiantado tienen que colaborar para dar una respuesta que integre decisiones metodológicas y tecnológicas y, a la vez, garantice la equidad, la seguridad jurídica y la transparencia para todos los actores, internos y externos. Por consiguiente, presentan una guía de recomendaciones para ayudar al profesorado y a las universidades en este proceso educativo tomando en cuenta la evaluación continua de las asignaturas, la opción de prescindir totalmente de las pruebas finales, la garantía del máximo nivel de identificación de los estudiantes, el uso de tecnologías ya disponibles en la universidad, la entrega de trabajos, ejercicios, infografías, la habilitación de sistemas de evaluación por pares, la solicitud de presentaciones o defensas de los trabajos usando las herramientas de videoconferencia con una duración limitada, la realización de pruebas orales individuales, la consideración de la casuística del estudiantado y la incorporación de soluciones de e-proctoring.

En el caso de México, el Estado Mexicano señala que existe evidencia de un sector educativo con cargas de trabajo y procesos evaluativos que carecen de sentido, es decir, en este contexto de pandemia, se observan limitantes para lograr un proceso educativo acorde con las necesidades de los estudiantes (Álvarez, 2020). Asimismo, es un momento que obliga a repensar el sentido de la institución escolar y del currículo formal, de los contenidos disciplinarios alejados de la realidad, de las prácticas docentes hegemónicas, y de la gestión académico-administrativa atada a reglamentos obsoletos. La escuela como la conocemos deberá cambiar para asumir los retos que planteará la sociedad que surja de esta contingencia sanitaria, de la inminente crisis económica que posiblemente modifique los procesos productivos de nuestra fábrica global y de la experiencia adquirida de manera forzada en torno al uso de las TIC en educación (Barrón, 2020)

Por otra parte, la pandemia de covid-19 ha puesto de manifiesto las carencias de las instituciones de educación superior en materia de infraestructura y de formación del personal académico para llevar a cabo, de manera satisfactoria, la educación en línea. También ha exhibido de manera clara las enormes desigualdades que existen entre la población estudiantil, las cuales hacen temer que la brecha digital y la del aprendizaje se puedan seguir ensanchando (Alcántara, 2020). En México encontramos estas inequidades y mucho retraso en las inversiones físicas en las escuelas, la conectividad de banda ancha, el equipamiento, el software y la formación de los trabajadores de la educación en esta materia. Las posibilidades de trabajo sincrónico entre maestros y estudiantes, el número y tipo de recursos tecnológicos utilizados, o las condiciones para dar marcha a la educación digital hacen evidente las diferencias entre modalidades y tipos educativos, escuelas privadas y escuelas públicas, entre el medio rural y el urbano, entre zonas industrializadas y de mayoría indígena. Estas diferencias potencian la exclusión y el rezago educativos, obstaculizan el ejercicio ciudadano de la libertad y de la democracia, y mantienen el círculo de la pobreza y la inequidad (Chehaibar, 2020). 
Dado que la emergencia sanitaria no ha terminado, no es tiempo todavía de hacer un balance de los daños ni de las estrategias que se tendrán que desarrollar para recuperar lo perdido, principalmente en términos de los avances en el aprendizaje de los alumnos. Es posible, adelantar que lo irregular de la situación en la que algunas instituciones pudieron trabajar en línea de mejor modo que otras, habrá de representar un enorme problema para reducir los desequilibrios ocurridos durante este periodo, una vez que se regrese a las aulas (Alcántara, 2020)

En el caso Costa Rica, en el contexto de la pandemia, se pone en evidencia las diferencias marcadas que presenta la educación privada en relación con la educación pública, Guardia (2020) señala que, en cuanto a la implementación de la educación a una modalidad virtual, se hace presente la brecha digital que se presenta en las zonas periféricas en comparación con lo que ocurre en el centro del país. Igualmente, indica la importancia que tiene el espacio del centro educativo, ya que muchos estudiantes en sus hogares no cuentan con los espacios adecuados para reinventar el desarrollo de los contenidos, además de la proporción de alimentos que obtiene el estudiantado en el centro educativo. En el sistema educativo costarricense la alimentación se da en todos los niveles, y en el nivel universitario, se brinda residencia para los estudiantes en condición de vulnerabilidad, no obstante, ante la crisis sanitaria al pasar a una modalidad virtual, muchos estudiantes han regresado a sus hogares indicando graves problemas de continuidad en sus estudios universitarios.

Para el desarrollo de la investigación se establecen objetivos que guían el estudio, considerando la revisión bibliográfica, el trabajo de campo y el análisis de los datos, para tener resultados válidos y confiables; en este sentido, los objetivos estipulados refieren a explicar los cambios experimentados en los procesos de enseñanza y aprendizaje al pasar de una modalidad presencial a una modalidad virtual; identificar la forma en que los docentes se refieren al cambio en la impartición de las lecciones virtuales; distinguir las actividades y las expectativas de los docentes sobre los estudiantes en la virtualidad y el rol que ha desempeñado en el contexto virtual.

\section{Método}

El método establecido en esta investigación es la Teoría Fundamentada debido a que se justifica su selección por la necesidad de realizar un estudio en el cual se pueda explicar el objeto de investigación. En este sentido, se considera la fidelidad de la propia expresión de los participantes y el mantenimiento del significado que tienen las palabras para sus propios protagonistas, pues se busca "patrones repetidos de acontecimientos, sucesos, o acciones/interacciones que representen lo que las personas dicen o hacen, solas o en compañía, en respuesta a los problemas y situaciones en los que se encuentran" (Corbin y Strauss, 2002, p. 142). Es decir, mediante este método se logra establecer códigos que permiten realizar una aproximación teórica sobre el fenómeno en estudio, el cual no se ha profundizado en otras investigaciones; en este caso, se trabaja en la explicación de aspectos que se generan a partir de un cambió que ha impactado los procesos de enseñanza y aprendizaje, al pasar de una modalidad presencial a una virtual.

\subsection{Participantes}

Los participantes del estudio son docentes del contexto educativo universitario de México y Costa Rica que, debido a la crisis sanitaria, pasaron a impartir lecciones en la modalidad virtual, una vez que ya había iniciado el ciclo lectivo en la modalidad presencial; por lo tanto, se seleccionan a docentes que tengan esta condición para obtener datos de cómo se han adaptado desde su rol docente a estos cambios enfrentados. En total, 
participaron doce docentes que imparten clases en los centros educativos en los que se realiza el estudio. Se trata de profesores con más de 18 años de servicio docente, en la modalidad presencial, que han utilizado de forma parcial las tecnologías durante el desarrollo de las lecciones; esta selección de los participantes permite visualizar que al no tener experiencia suficiente en el uso de las TIC, y a raíz de la pandemia por la enfermedad de la COVID-19, tuvieron que enfrentarse a un nuevo entorno virtual para desarrollar su quehacer docente, $y$ desde ese momento hacer un replanteamiento de su posición en un espacio poco conocido para culminar un curso lectivo según las habilidades que fueran adquiriendo en relación con las herramientas y el uso que se debe de hacer de las TIC.

\subsection{Diseño}

El diseño metodológico se realiza desde la Teoría Fundamenta, debido a que es una metodología de orden cualitativo que permite contextualizar los fenómenos que se estudian, lo cual tiene particular aceptación cuando se requiere comprender y explicar el tema de investigación. Por ende, el uso de la teoría fundamenta, en este caso, aporta elementos para indagar y entender lo que se suscita en el cambio de modalidad de los docentes al impartir lecciones en el ámbito virtual, con nuevas experiencias en el proceso educativo universitario. Es así, como al considerar el distanciamiento social que se requiere en este momento de crisis, se optó por realizar las entrevistas en un medio virtual, seleccionando la plataforma Zoom y Meet. En este proceso las respuestas fueron grabadas y transcritas línea por línea, de manera que con esto se fueran teniendo evidencias de las propias palabras de los participantes (Corbin y Strauss, 2002).

\subsection{Instrumento}

La técnica utilizada en la investigación es la entrevista en profundidad, la cual se aplicó a los participantes seleccionados en este estudio. Para la entrevista se realizó una guía de preguntas, en dos ámbitos temáticos relacionados con el cambio en el rol docente en la modalidad virtual; en primer lugar, se enfocó en el tema de la función docente en el espacio virtual, en el que se ahondó sobre el cambio experimentado al pasar de las lecciones presenciales a las lecciones virtuales, también se indagó acerca de las adecuaciones realizadas en los cursos impartidos y en las funciones adicionales que realizan los docentes para adaptarse a este cambio; aquí destaca el proceso de actividades llevadas a cabo, el progreso de los estudiantes y la evaluación que se utiliza. Fue relevante analizar desde la percepción docente en cuanto a las expectativas y su rol fundamental en el contexto virtual. En segundo lugar, se profundizó, en cuanto a la relación con estudiantes en el espacio virtual, donde se destaca el seguimiento a la población estudiantil, la forma en que se registra la comprensión de los contenidos desarrollados y la comunicación que se genera en la modalidad virtual.

\subsection{Procedimiento}

En el procedimiento para llevar a cabo la investigación, se establecieron tres fases: la primera fase implicó la elaboración de la guía de preguntas que responde a la técnica de la entrevista en profundidad. La segunda fase consistió en la validación del instrumento por expertos, de manera que esto permitiera que la guía de preguntas estuviera clara con el tema relacionado. La tercera fase consistió en la aplicación del instrumento a los participantes del estudio. La aplicación se siguió desde la implementación de la interacción profesional mediante un acercamiento basado en el interrogatorio cuidadoso y la escucha, con el propósito de obtener conocimiento meticulosamente comprobados. (Kvale, 2011). 


\section{Resultados}

El procedimiento del análisis de los datos siguió los postulados de Corbin y Strauss (2002), tal y como se consignan en la figura 1. de manera que con ellas se alcanzaron los resultados en cada una de estas etapas: la codificación abierta, axial, selectiva y la conformación de una matriz condicional. Para ello se establecen respuestas en cada uno de los objetivos del estudio.

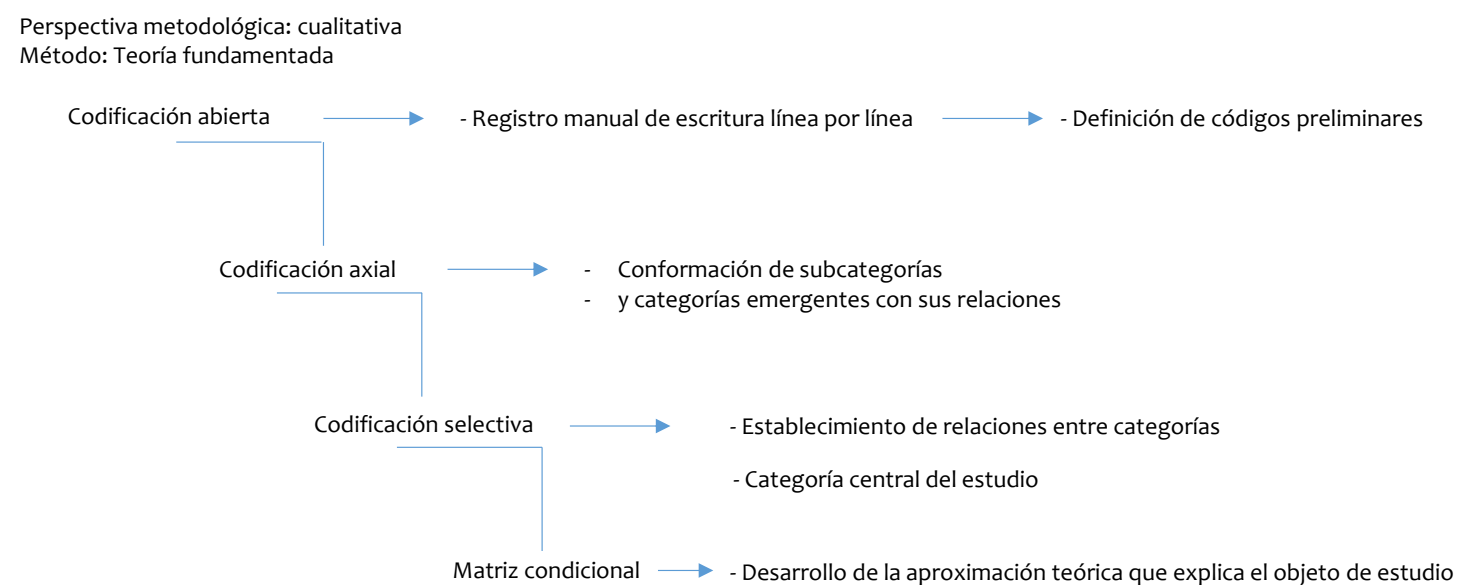

Figura 1. Tratamiento analítico de la información

\subsection{Codificación abierta}

En la codificación abierta, se realizó un registro manual de la escritura línea por línea de los datos obtenidos, en esto, se consideraron manifestaciones, palabras y frases de los participantes y, por último, se definieron los códigos preliminares. Tabla 1.

Tabla 1.

Códigos preliminares

Códigos preliminares

\begin{tabular}{|c|c|}
\hline Rol docente & $\begin{array}{l}\text { Se ha tenido que ser creativo y facilitador en cuanto a favorecer } \\
\text { los espacios de encuentro para el aprendizaje. }\end{array}$ \\
\hline $\begin{array}{l}\text { Expectativa de los } \\
\text { docentes sobre los } \\
\text { estudiantes en la } \\
\text { virtualidad }\end{array}$ & $\begin{array}{l}\text { Se destaca que el estudiante debe tener más autonomía, ya que } \\
\text { en un contexto de presencialidad, el docente está más pendiente } \\
\text { de la mediación de los contenidos. }\end{array}$ \\
\hline Presencialidad & $\begin{array}{l}\text { Se dan manifestaciones que requieren mayor presencia de } \\
\text { estudiantes en el desarrollo visual de las lecciones. }\end{array}$ \\
\hline $\begin{array}{l}\text { Relación } \\
\text { interpersonal }\end{array}$ & $\begin{array}{l}\text { Se indica que en el contexto virtual es poco viable desarrollar los } \\
\text { lazos con los estudiantes. }\end{array}$ \\
\hline Profesor virtual & $\begin{array}{l}\text { Se señalan como relevante la existencia de una relación distante } \\
\text { y poca presencia de técnicas utilizadas. }\end{array}$ \\
\hline Virtualidad & $\begin{array}{l}\text { Se genera un retroceso al tener que priorizar los contenidos que } \\
\text { se llevan a cabo en las sesiones virtuales. }\end{array}$ \\
\hline Tecnología & $\begin{array}{l}\text { Se expone que se debe avanzar a mecanismos que permitan una } \\
\text { mayor equidad en cuanto al aprendizaje sincrónico y asincrónico. }\end{array}$ \\
\hline
\end{tabular}




\begin{tabular}{ll}
\hline $\begin{array}{l}\text { Acercamiento } \\
\text { humano }\end{array}$ & $\begin{array}{l}\text { Se recae en comunicados por medio de correos electrónicos y } \\
\text { mensajes de texto por WhatsApp, que no permiten tener un } \\
\text { acercamiento con los estudiantes. }\end{array}$ \\
\hline Actividades & $\begin{array}{l}\text { Se señala que no hay certeza de saber si con ellas se logra el } \\
\text { aprendizaje y profundización de los temas por parte de los } \\
\text { estudiantes. }\end{array}$ \\
\hline Docente presencial & $\begin{array}{l}\text { Se enfatiza que lo presencial no es sustituible y que en lo virtual } \\
\text { hay mucho más trabajo para el tratamiento de los temas. }\end{array}$ \\
\hline
\end{tabular}

Elaboración propia (2020)

\subsection{Codificación axial}

Una vez pasada la etapa de la codificación abierta con las características propias, de los códigos preliminares, se procedió a la segunda etapa que fue la codificación axial, en este proceso se estableció la conformación de las subcategorías y las categorías emergentes, (Tabla 2). Las cuales permiten realizar un proceso de relaciones entre ellas para, posteriormente, en la codificación selectiva plantear una categoría central del estudio.

Tabla 2.

Categorías emergentes y subcategorías

\begin{tabular}{|c|c|}
\hline Categorías & Subcategorías \\
\hline $\begin{array}{l}\text { Requerimientos del rol del docente en el } \\
\text { proceso virtual de enseñanza }\end{array}$ & $\begin{array}{l}\text { Creatividad en el proceso } \\
\text { Facilitador de los contenidos } \\
\text { Acompañamiento al estudiantado } \\
\text { Espacios de encuentro }\end{array}$ \\
\hline $\begin{array}{l}\text { Acciones realizadas en torno a las } \\
\text { tecnologías en el proceso educativo virtual }\end{array}$ & $\begin{array}{l}\text { Atención virtual } \\
\text { Seguimiento a estudiantes } \\
\text { Aprendizaje sincrónico } \\
\text { Conectividad }\end{array}$ \\
\hline $\begin{array}{l}\text { Presencialidad en el desarrollo virtual de } \\
\text { lecciones }\end{array}$ & $\begin{array}{l}\text { Presencia en los procesos de enseñanza y } \\
\text { aprendizaje virtual }\end{array}$ \\
\hline $\begin{array}{l}\text { Reivindicación de la presencialidad en los } \\
\text { procesos educativos }\end{array}$ & $\begin{array}{l}\text { Diferentes insumos en la presencialidad } \\
\text { Facilidad de aclaraciones } \\
\text { Mayor tiempo permite amplitud } \\
\text { Relaciones interpersonales }\end{array}$ \\
\hline Proceso virtual sin planificación & $\begin{array}{l}\text { Retroceso en la formación virtual } \\
\text { Proceso virtual no planificado } \\
\text { Mayor tiempo en las sesiones }\end{array}$ \\
\hline Recargo de labores en el proceso virtual & $\begin{array}{l}\text { Proceso de mayor tiempo } \\
\text { Cambio de tiempo y espacio } \\
\text { Multiplicidad de tareas docente }\end{array}$ \\
\hline
\end{tabular}

Elaboración propia (2020)

\subsection{Codificación selectiva}

La tercera etapa fue la codificación selectiva, la cual permitió establecer las relaciones entre las categorías generando una aproximación a la categoría central del estudio, considerando las propiedades, las dimensiones y relaciones surgidas durante el análisis realizado. Es así, como a partir de las categorías emergentes y las subcategorías, se puede establecer que, en los procesos educativos virtuales, se establecen condiciones 
paradigmáticas que deben "permitirle acceder al conocimiento del deber ser para el saber hacer para un desarrollo de la inteligencia y que le permita al ser humano prepararse para esperar lo inesperado y saber afrontarlo" (Yepes, 2019, p, 10). Es decir, resulta indispensable considerar que los cambios que se presentan al pasar de la presencialidad a la virtualidad como forma de enseñar, deben generar una nueva forma de pensar las relaciones por parte de los docentes con sus estudiantes, por lo que deben procurar el alcance de un replanteamiento y la resignificación asumiendo con responsabilidad los retos que se imponen en este nuevo panorama de cambios en los espacios educativos virtuales.

\subsection{Matriz condicional}

El proceso que conlleva el establecimiento de una matriz condicional permite evidenciar cómo se ubica el fenómeno estudiado en su contexto, de manera que se relatan las relaciones que surgen en este proceso de incertidumbre al pasar a una modalidad virtual y dejar atrás el proceso de la presencialidad. De esta forma, los docentes indican nuevos requerimientos del rol docente en el proceso virtual de enseñanza, para el cual deben establecer acciones que procuren entender dichos elementos y que le permitan entablar de manera asertiva la presencialidad en los espacios virtuales, para reivindicar que ante todo, lo indispensable es establecer un trabajo virtual que no contiene el respaldo de una adecuada planificación para desarrollar actividades didácticas, metodológicas y curriculares que respondan al estudiantado y les motive a participar de las lecciones.

Es así, que esta visualización de mayores labores no sea un elemento que repercuta en un proceso educativo en el que no se alcance la meta de tal proceso. Por tanto, se hace indispensable que las instituciones involucradas para gestionar los espacios virtuales deben establecer estrategias de formación de los docentes para insertarse y seguir de manera adecuada en el proceso virtual que es inminente en este contexto actual, donde el respaldo de la seguridad de las personas es fundamental, y así seguir con los procesos de enseñanza y aprendizaje en el que la creatividad sea el motor de respuesta al desarrollo de los temas.

En relación con el objetivo de explicar los cambios experimentados en los procesos de enseñanza y aprendizaje al pasar de una modalidad presencial a una modalidad virtual, los docentes indican que en la presencialidad hay cuestiones que son insustituibles para realizar el proceso de educativo; asimismo, señalan que son un sinnúmero de cuestiones que pueden hacer en la presencialidad y en la virtualidad no, aunque lo intenten, y tengan que integrar muchos elementos para tratar de desarrollar los contenidos. No hay sustituto alguno para ello, y es esto lo que se tiene que rescatar, ahora, en tiempos de pandemia: precisamente esa presencialidad que ha permitido, durante siglos, la formación de calidad de los profesionistas. El Cambio es bastante difícil, dado que en lecciones presenciales todos o casi todos los alumnos están en el aula, al contrario, en las clases remotas, en donde solo se conecta cuando mucho la mitad del grupo, y existe muy poca interactividad entre el profesor y los estudiantes.

No obstante, los profesores señalan que llevar a cabo las lecciones en otra modalidad es un proceso nuevo del que hay que aprender e ir creando mecanismos y soluciones para convertir la virtualidad en una realidad en el proceso formativo. En la virtualidad el docente debe crear contenido didáctico para realizar la mediación, porque ese material didáctico debe ser lo suficientemente claro y completo para que, cuando la persona participante lo estudie pueda comprenderlo sin la explicación de la persona docente.

De igual manera, los educadores indican que el mundo está cambiando muy rápido, y que debido a la situación actual estamos adelantando 10 años el uso e implementación de herramientas para la educación y los negocios, y que las nuevas generaciones tienen más 
capacidades para el autoaprendizaje, son más adaptables a entornos virtuales y cada día tienen más acceso a información

En relación con el objetivo de identificar la forma en que los docentes se refieren al cambio en la impartición de las lecciones virtuales, estos señalan que hay que avanzar hacia mecanismos que permitan una mayor equidad en cuanto al aprendizaje sincrónico, que cada día más estudiantes puedan acceder al conocimiento sin tantas dificultades en el acceso tecnológico. También, manifiestan su descontento por la falta de participación del estudiantado en algunas clases; indican que están muy acostumbrados a que el maestro lleve el liderazgo de la clase y que sea el que más hace en la clase. Sin embargo, perciben que como docentes deben conjuntar conocimientos, experiencia, creatividad y tecnología para ayudar a construir los conocimientos de los alumnos.

Asimismo, uno de los cambios que manifiestan sentir es que para impartir lecciones en la virtualidad requieren de más tiempo y de una preparación mejor en el uso de las tecnologías que, por la situación del cambio abrupto al uso de las TIC, no tienen el conocimiento y la práctica de dichas tecnologías en las clases presenciales.

En la búsqueda de distinguir las actividades, las expectativas de los docentes sobre los estudiantes en la virtualidad y el rol que ha desempeñado en el contexto de la virtualidad manifiestan que en los trabajos incluyen preguntas para determinar el grado de alcance de los contenidos, pero si los estudiantes no lo remiten no hay información. No se tiene la certeza de que estén logrando las habilidades esperadas ya que, mediante la observación de las participaciones y aportes, en las diferentes actividades propuestas, sean sincrónicas o asincrónicas, no se evidencia que los estudiantes respondan y muy pocos envían sus trabajos.

Además, manifiestan que hubo un cambio en la cantidad de actividades de evaluación, porque en la virtualidad el estudiantado requiere espacios para la lectura e interiorización, así que han disminuido las actividades evaluativas. Igualmente, cambiaron en cuanto a desarrollar actividades muy puntuales a lo que "se necesita o espera" que logren, dejando de lado actividades que permitían un conocimiento adicional o más amplio de los diversos temas como se hace en la presencialidad.

Los profesores muestran que las expectativas de la modalidad virtual son muy altas, principalmente porque tenemos como principal herramienta el Internet y su enorme capacidad de información que puede proporcionar; para esta modalidad el docente debe tener un mayor seguimiento por cada estudiante, dar continuidad y motivación para el cumplimiento de las actividades, tener una mayor comunicación a través de redes sociales. Se favorecen espacios de encuentro para el aprendizaje, que sean retadores, motivantes y contextuales para la situación social que se está viviendo, donde se respete el ritmo personal de cada participante. Asimismo, los docentes señalan que la expectativa es que después de vacaciones y al ver la dificultad de volver a la presencialidad los estudiantes se hagan más presentes en el desarrollo virtual de las lecciones.

En relación con el rol docente, la percepción que manifiestan es que son creadores de contenidos y que tienen la tarea de realizar ambientes de aprendizaje, en el que guíe a sus estudiantes para acceder al conocimiento. Asimismo, señalan que el rol debe ser activo, facilitador de los contenidos hacia los estudiantes por medio de la contextualización y la evaluación constante. El rol es más de un facilitador que de un educador y formador, puesto que se deja de lado estos papeles que son los que forman la integridad del estudiante; dichos aspectos no son considerados en la virtualidad

También en cuanto al rol en la virtualidad, los docentes indican que deben ser capaces de despertar las capacidades de análisis con la ayuda de instrumentos digitales y adaptarlas 
para cada estilo de aprendizaje que pueden presentar los alumnos. De igual forma, debe ser un experto en el uso de las Tecnologías de la Información y la Comunicación, ya que debe ser capaz de saber usar la herramienta digital para el tipo de conocimiento a generar.

\section{Discusión y conclusiones}

Los hallazgos de la investigación permiten alcanzar el objetivo propuesto, ya que se explica que el cambio de una modalidad presencial de lecciones a una modalidad virtual ha sido un proceso en el que no se ha manifestado una planificación ordenada, debido a la premura de la impartición de lecciones en la nueva modalidad.

En este sentido, hay evidencia que se está ante una nueva realidad de la que hay que aprender y crear mecanismos y soluciones para convertir la virtualidad en el proceso educativo, por lo que en este panorama el docente debe establecer métodos alternativos para el desarrollo del material didáctico, en procura de que las acciones para impartir lecciones sean claras manteniendo un ambiente que propicie la participación del estudiantado. En relación con los elementos encontrados en esta investigación hay consenso con lo que establece la Unesco (2020), al identificar desde los participantes que los docentes no estaban preparados para enfrentar estos cambios y que las personas con menos recursos tecnológicos se convierten en la población estudiantil más vulnerable, debido a que tienen menor participación y presencia en los procesos de enseñanza y aprendizaje virtual, ya que no cuentan con las herramientas necesarias para ser parte de lo que se lleva a cabo en las lecciones desde la virtualidad.

Los docentes expresan que es mucho más trabajo, debido a las funciones agregadas, es decir, todas las actividades docentes se realizan muchas veces más (se revisa más, se retroalimenta más, se sustituye la explicación verbal por la explicación escrita y eso hace que el trabajo se vaya multiplicando por la cantidad de alumnos que tenga); los docentes vivencian experiencias de angustia y cansancio al hacer un replanteamiento de actividades para llegar a toda la población estudiantil, no obstante, que lo que priva ante todo es el derecho del estudiantado a recibir las lecciones de forma pertinente, por lo cual se da seguimiento de manera grupal e individual, mediante el uso de plataformas como Zoom, Meet y WhatsApp, de manera que el seguimiento sea oportuno y suficiente.

Del mismo modo, referente a las evaluaciones a nivel universitario, se concuerda en que en este contexto se debe dar un cambio y transformación en los procesos virtuales, es así como "la evaluación se une a la falta de experiencia institucional en este ámbito, pues no se han practicado ni probado estos procesos de forma no presencial (García, Corell, Abella y Grande, 2020 p. 12). Es decir, tal y como se explica en la investigación realizada no existe un instrumento de evaluación virtual que permita certificar el aprendizaje.

En este devenir de cambio de lo presencial a lo virtual, el rol del docente destaca características de ser un facilitador, creativo y acompañante para continuar con las actividades que permitan avanzar hacia mecanismos que hagan posible una mayor equidad en cuanto al aprendizaje sincrónico, para que se pueda acceder al conocimiento sin tantas dificultades en el acceso tecnológico. Así, los docentes señalan que es indispensable que se favorezcan encuentros para el aprendizaje que sean retadores, motivantes y contextualizados en el que se respete el ritmo personal de cada participante.

Al igual que lo plantea Moreno (2020) se coincide en cuanto a replantear los procesos de enseñanza y aprendizaje, de manera que en este accionar, se utilicen metodologías para establecer un privilegio del aprendizaje, por encima del mismo proceso de enseñanza, resaltando el papel de las TIC; en efecto, los hallazgos de la investigación señalan que existe 
una estrecha relación con ese planteamiento, pues los docentes indican que es un proceso nuevo del que hay que aprender, crear mecanismos y soluciones para convertir la virtualidad en una realidad en el proceso educativo. Esto hace que los docentes en esta nueva realidad tengan que conjuntar conocimientos, experiencia, creatividad y tecnología para ayudar a construir los saberes de los estudiantes.

Los hallazgos permiten establecer la realidad que los docentes requieren una formación continua en el uso de las tecnologías, tanto para ser utilizadas en las clases presenciales y ante todo en la modalidad virtual, es así como el docente debe mantenerse dirigido a un trabajador de campo que pueda responder adecuadamente a las carencias que tienen los estudiantes para participar en la modalidad virtual.

Por tanto, es inminente una resignificación del rol docente universitario a partir de una crisis sanitaria, es por esto que, en el cambio de lo presencial a lo virtual, los docentes deben actualizarse en el uso de las TIC, en la capacitación de la implementación de cursos por plataformas digitales, en procesos de evaluaciones digitales y la continuidad que requieren los estudiantes en el proceso educativo. Se requiere que se les dé un sentido diferente a los procesos de enseñanza y aprendizaje tradicionales, para dar una nueva comprensión de lo que ocurre en la actualidad, es decir, se requiere dar una interpretación diferente a lo que se ha gestado en los procesos educativos en la educación presencial.

Por ello, la categoría central, establece la resignificación del rol del docente como un elemento fundamental para que su reformulación y su aprendizaje en acciones virtuales puedan responder de forma asertiva y sea un aporte en la construcción de los aprendizajes de la población estudiantil. En síntesis, esta resignificación del rol del docente en los procesos virtuales, debe responder a las realidades de los que concurren en este espacio, en procura de alcanzar las metas educativas con un rol activo, creativo y afectivo en respuesta a la población estudiantil que convergen en las lecciones de forma virtual, de ahí que la resignificación del rol docente, requiere de un proceso en el que converja una capacitación y la disponibilidad de los recursos para una planificación acorde al contexto de virtualización de las lecciones.

Los cambios que han ocurrido en las prácticas de aulas en tiempos de crisis al asumir otra modalidad de impartición de lecciones, conlleva a que los docentes, realicen una planificación adecuada para el aprovechamiento del tiempo, por ello, deben actualizarse en cuanto a ver cuáles son las herramientas virtuales que mejoren los procesos de enseñanza y aprendizaje. Igualmente, se debe trabajar en la búsqueda de que las actividades deban responder a la población estudiantil, de ahí que los administradores de los centros educativos deben generar espacios para que los docentes adquieran las habilidades para la elaboración de actividades, procesos de evaluación y se trabaje en materiales oportunos y de calidad para alcanzar un trabajo idóneo en la virtualidad.

\section{Referencias}

Alcántara-Santuario, A. (2020). Educación superior y COVID-19: una perspectiva comparada. En Educación y pandemia, una visión académica. (pp. 75-82). Universidad Nacional Autónoma de México.

Álvarez-Mendiola, G. (2020). Covid-19. Cambiar de paradigma educativo. Consejo Mexicano de Investigación Educativa. http://www.comie.org.mx/v5/sitio/2020/04/16/covid-19cambiar-de-paradigma-educativo/ 
Barrón-Tirado, M. C. (2020). La educación en línea. Transiciones y disrupciones. En Educación y pandemia, una visión académica. (pp. 66-74). Universidad Nacional Autónoma de México.

Britez, M. (2020). La educación ante el avance del COVID-19 en Paraguay. Comparativo con países de la Triple Frontera. Universidad Nacional del Este de Paraguay. https://doi.org/10.1590/SciELOPreprints.22

Chehaibar, M. L. (2020). Flexibilidad curricular. Tensiones en tiempos de pandemia. En Educación y pandemia, una visión académica. (pp. 83-91). Universidad Nacional Autónoma de México.

Corbin, J., y Strauss, A. (2002). Bases de la investigación cualitativa. Técnicas y procedimientos para desarrollar la teoría fundamentada. Universidad de Antioquía.

Diario Oficial de la Federación (2020). Acuerdo por el que se establecen acciones extraordinarias para atender la emergencia sanitaria generada por el virus SARSCoV2. Gobierno de México. https://www.dof.gob.mx/nota_detalle.php?codigo=5590914\&fecha=31/03/2020\&pri nt=true

García-Peñalvo, F. J., Corell-Almuzara, A., Abella-García, V., \& Grande-de Prado, M. (2020). Online Assessment in Higher Education in the Time of COVID-19. Education in the Knowledge Society, 21(12),1-12 . doi:10.14201/eks.23013

Guardia-Donato, Diana. (2020, mayo). ¿Qué pasa con la infancia costarricense durante la cuarentena? Semanario Universidad. https://semanariouniversidad.com/opinion/quepasa-con-la-infancia-costarricense-durante-la-cuarentena/?

Hodges, C., Moore, S., Lockee, B., Trus, T. y Bond, A. (2020). The Difference Between Emergency Remote Teaching and Online. EDUCAUSErevieu. https://er.educause.edu/articles/2020/3/the-difference-between-emergency-remoteteaching-and-online-learning

Kvale, S. (2011). La entrevista en Investigación Cualitativa. Ediciones Morata, S. L.

Moreno-Correa, S. (2020). La innovación educativa en los tiempos del Coronavirus. Salutem Scientia Spiritus, 6( 1), 15-21. https://www.researchgate.net/publication/340515328

Organización de las Naciones Unidas para la Educación, la Ciencia y la Cultura. (2020, 07 de julio). Consecuencia del cierre de las escuelas. https://es.unesco.org/covid19/educationresponse

UNESCO. (2020, 18 de junio). Reconstruir mejor: tras el COVID-19, la educación debe cambiar para responder a la crisis climática. https://es.unesco.org/news/reconstruir-mejorcovid-19-educacion-debe-cambiar-responder-crisis-climatica

Yepes-Londoño, J. (2019). Sinopsis Los siete saberes para la educación del Futuro. ResearchGate.

https://www.researchgate.net/publication/332655292_Sinopsis_Los_siete_saberes para_la_educacion_del_Futuro 